\title{
Knuckle wire technique in percutaneous coronary intervention of chronic total occlusion
}

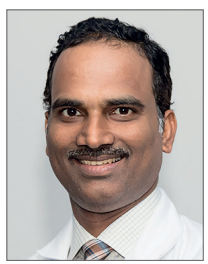

Sharath A. Reddy ${ }^{1 *}$, MD, DM, FSCAI, FACC, FAHA; Ajith Ananthakrishna Pillai², MD, DM; Bharath Reddy ${ }^{1}, \mathrm{MD}$, DM; Venkat Rao ${ }^{1}$, MD, DM; Aparajita Deshpande ${ }^{3}$, Pharm. D.

1. Department of Cardiology, Medicover Hospitals, Madhapur, Hyderabad, Telangana, India; 2. Department of Cardiology, JIPMER, Dhanvantri Nagar, Gorimedu, Puducherry, India; 3. Department of Research, Medicover Hospitals, Madhapur, Hyderabad, Telangana, India

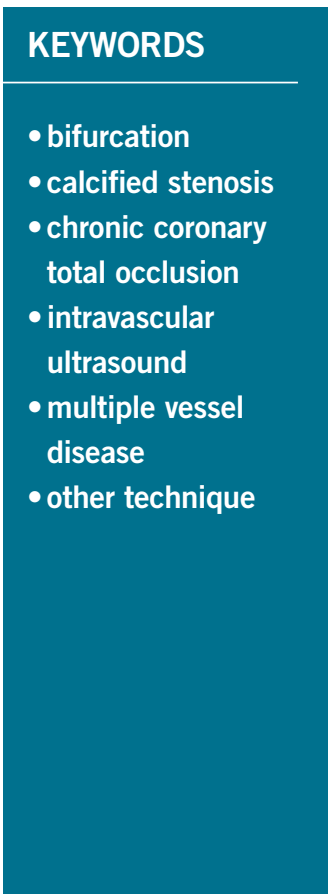

\section{Abstract}

In percutaneous coronary intervention, the knuckle wire technique is one of the approaches to cross the long and ambiguous course of the occluded segment. However, this technique is generally used as a last alternative, when all other techniques fail. Although knuckle wiring expedites chronic total occlusion crossing, it can also complicate the percutaneous coronary intervention strategy irreversibly. Therefore, understanding the various aspects of the knuckle wire technique is a prerequisite in a chronic total occlusion setting. The authors herein intend to describe in detail the knuckle wire technique and its safe and effective approach in various chronic total occlusion wiring strategies, while befitting to the scope of a mainstream interventionist.

*Corresponding author: Department of Cardiology, Medicover Hospitals, Madhapur, Hyderabad 500081, Telangana, India. E-mail:drsr.research@gmail.com 


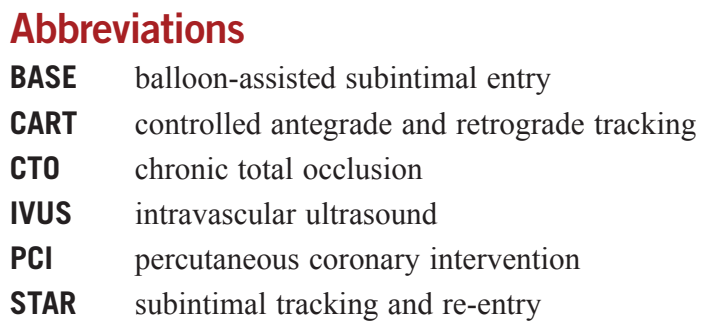

\section{Introduction}

The knuckle wire technique was designed for subintimal angioplasty originating in the peripheral circulation, having been first described in 1989 in percutaneous intervention for femoropopliteal occlusions. The original subintimal tracking and re-entry (STAR) technique with a knuckle wire was attempted by Colombo et al in coronary occlusions ${ }^{1}$. It involved creating a cleavage in the subintimal plane by advancing a hydrophilic wire with a J-loop configuration to allow a blunt dissection between the anatomical planes of the vessel using the principle of differential longitudinal and tangential resistance (Figure 1 ) $^{2,3}$. In the STAR technique, the knuckle wire is used both for subintimal entry and to continue dissection to re-enter into the true lumen at bends in the vessel or branch points; however, re-entry to the true lumen is quite unpredictable and not controllable. Therefore, in contemporary chronic total occlusion (CTO) intervention scenarios (Figure 2), the knuckle wire is used mostly as a last resort only to create subintimal dissection, and reentry is assisted by CrossBoss ${ }^{\mathrm{TM}}$ catheter-Stingray ${ }^{\mathrm{TM}}$ LP system (Boston Scientific Corp., Marlborough, MA, USA), controlled antegrade and retrograde tracking (CART), or reverse $\mathrm{CART}^{4,5}$. The knuckle technique, when performed antegrade or retrograde, helps in long occlusions, especially with calcification, to maintain the guidewire position in the subintimal space within the vessel $\operatorname{architecture}^{6-9}$.

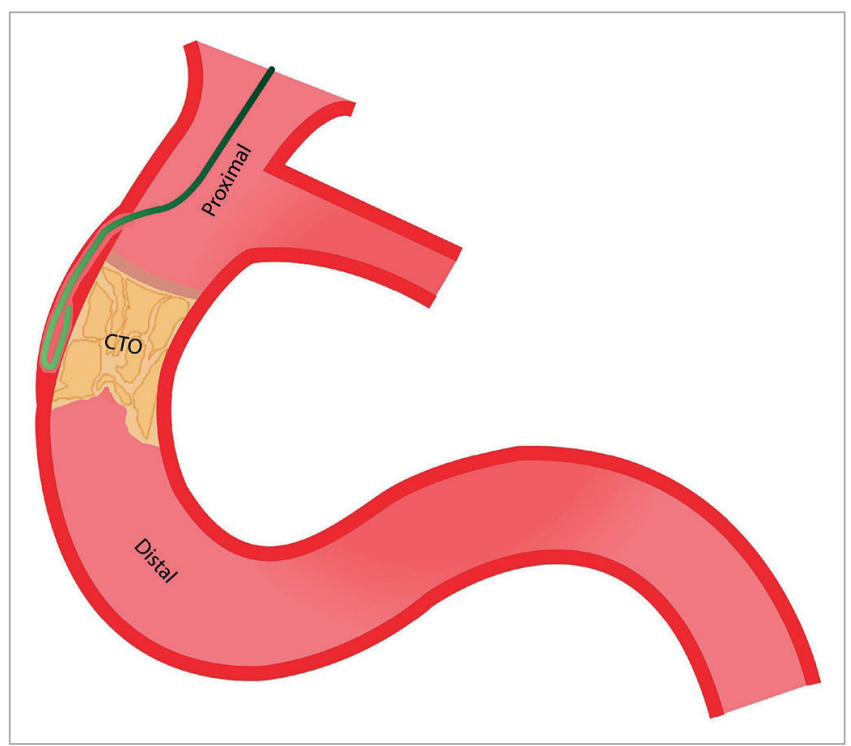

Figure 1. J-loop configuration of wire to allow a blunt dissection.

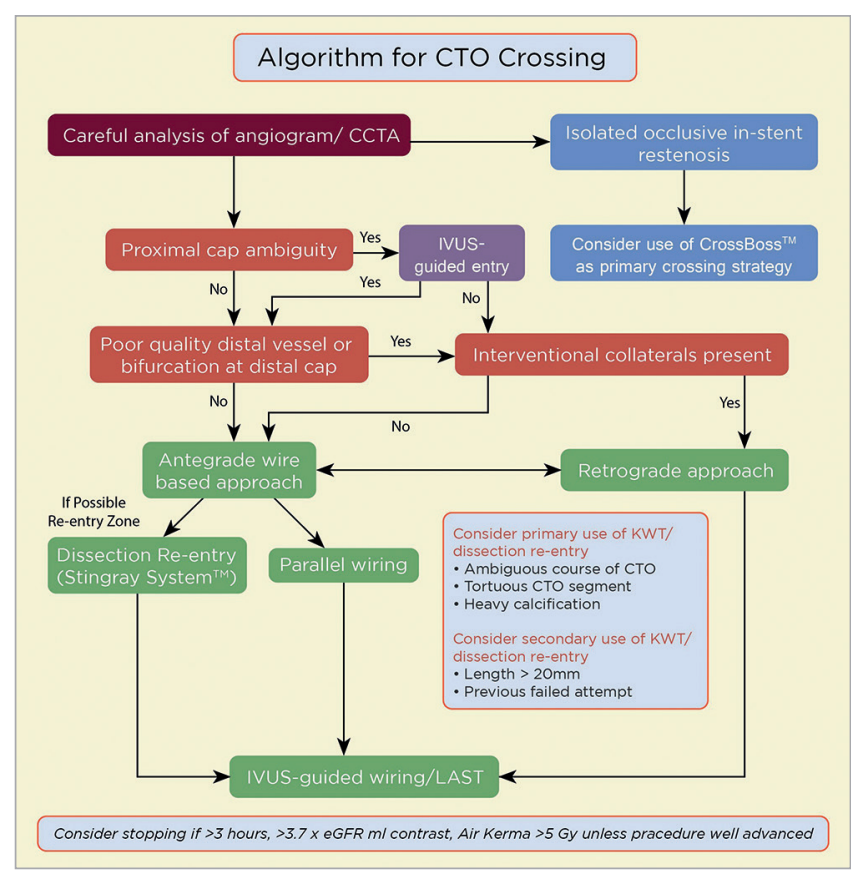

Figure 2. AP-CTO algorithm for CTO crossing. CCTA: coronary computed tomography angiography; CTO: chronic total occlusion; IVUS: intravenous ultrasound

\section{How the knuckle technique works: principle of differential resistance}

Knuckling works based on the principle of differential resistance of the subintimal space and media/adventitia tissue. The resistance offered by the subintimal space, i.e., longitudinal resistance, is less than the resistance presented by media/adventitia, which is the tangential/radial resistance. Therefore, when the knuckle wire is advanced, it moves down the subintimal low-resistance plane, rather than splitting-out through media/adventitia (Figure 3). As long as the knuckle width is within the size of the vessel, it remains in the subintimal space. If the width of knuckle exceeds the size of the vessel, it may cut through the media and perforate the vessel.

\section{Technique WIRES AND TIP SHAPING}

Polymer jacket hydrophilic wires with lower tip gram weight, such as the Fielder ${ }^{\mathrm{TM}}$ XT series (Asahi Intecc, Aichi, Japan), SION ${ }^{\circledR}$ black (Asahi Intecc), and the Pilot ${ }^{\mathbb{B}}$ series (Abbott Vascular, Santa Clara, CA, USA) are best suited for knuckle wiring. To create a knuckle, the wire must loop on itself such that the leading edge is usually the junction between the stiff shaft and the floppy distal segment (Figure 4B). The knuckle loop can be initiated by pre-shaping the wire tip as an "umbrella handle" and by applying forward pressure on the wire once it is within the CTO (Figure 4A). To avoid knot formation while forming a knuckle, the wire should not be rotated in any way. The advancement of a knuckle wire should always be done within an over-the-wire support microcatheter. This aids in controlling the knuckle width and length. Further, the microcatheter 


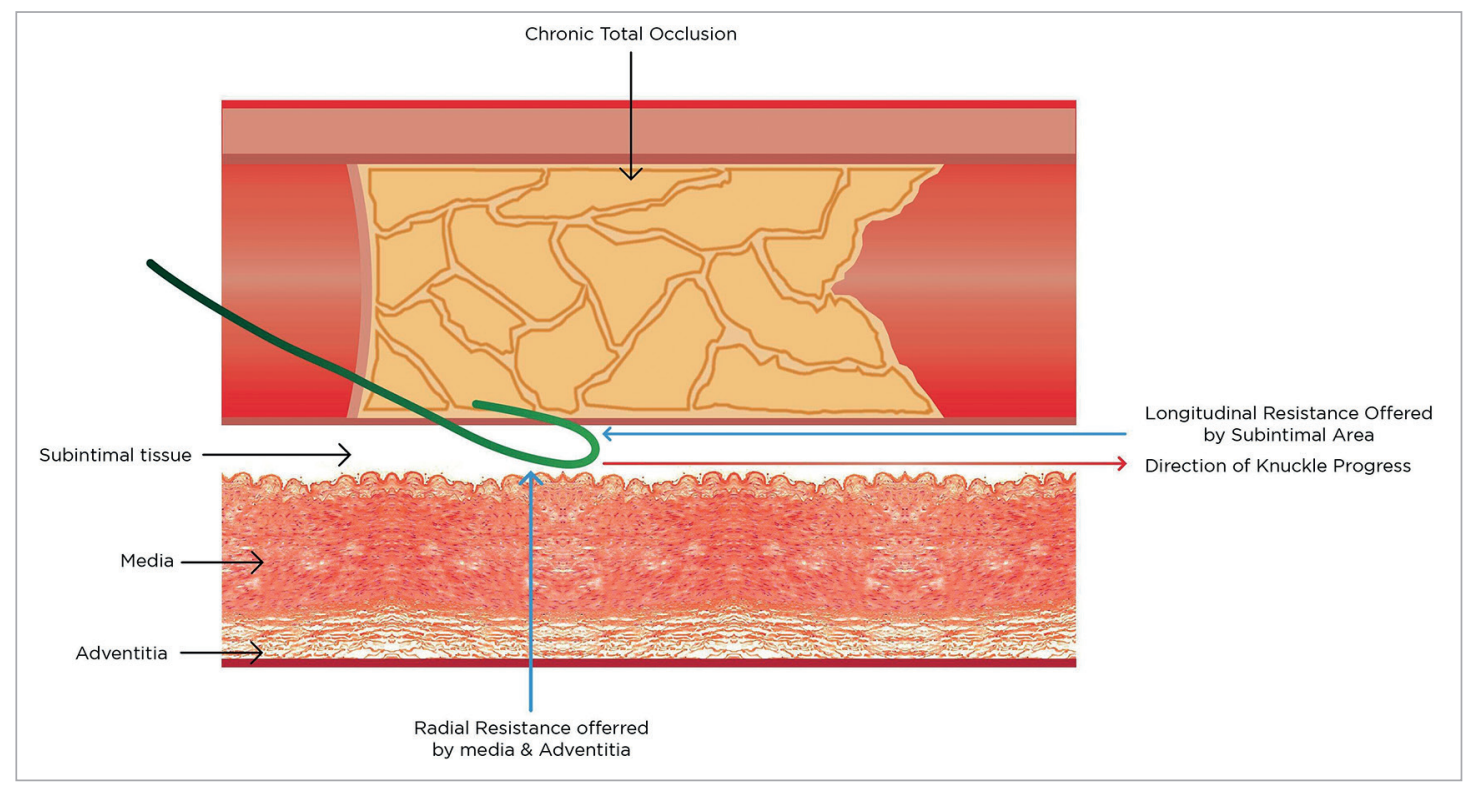

Figure 3. Differential resistance by subintimal tissue and media/adventitia tissue leads to knuckle progression in the subintimal plane.

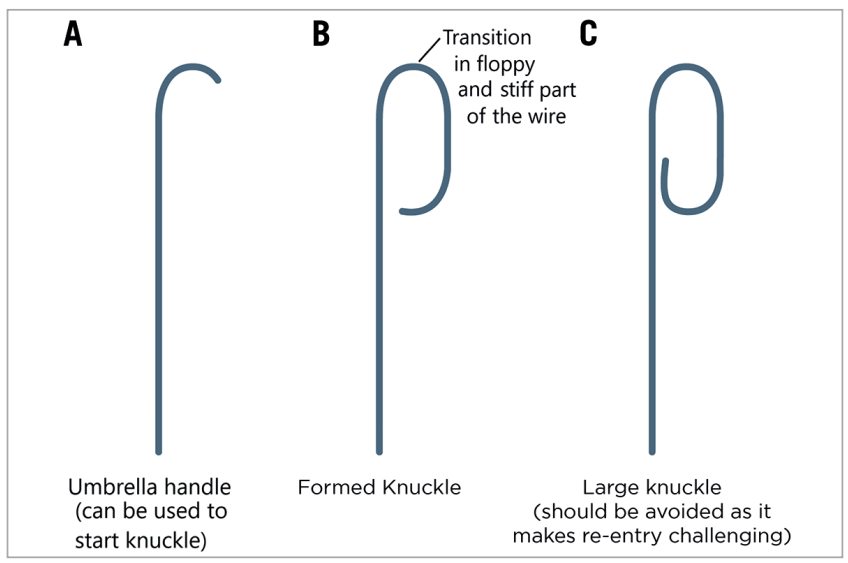

Figure 4. Wire and tip shaping. A) Umbrella handle, B) formed knuckle and C) large knuckle. aids in wire exchange if the wire gets trapped in calcified subintimal tissue, or gets broken. The microcatheter also helps in exchanging for a stiffer wire for true lumen re-entry once the knuckle crosses the CTO length. Once the knuckle is formed, further advancement of the knuckle should be performed keeping the microcatheter close to the tip of the knuckle ${ }^{10}$. Otherwise, the knuckle may shift onto the stiff portion of wire, leading to a wider knuckle which may split open the vessel (Figure 4C). Tip shaping is performed with a wide knuckle to avoid entry into side branches. The angiographic representation of tip shaping is shown in Figure $\mathbf{5}$.

\section{Where to start the knuckle? KNUCKLE WITHIN THE CTO SEGMENT}

Generally, it is not possible to enter the subintimal space without penetrating the proximal CTO cap first with a stiff wire or disrupting

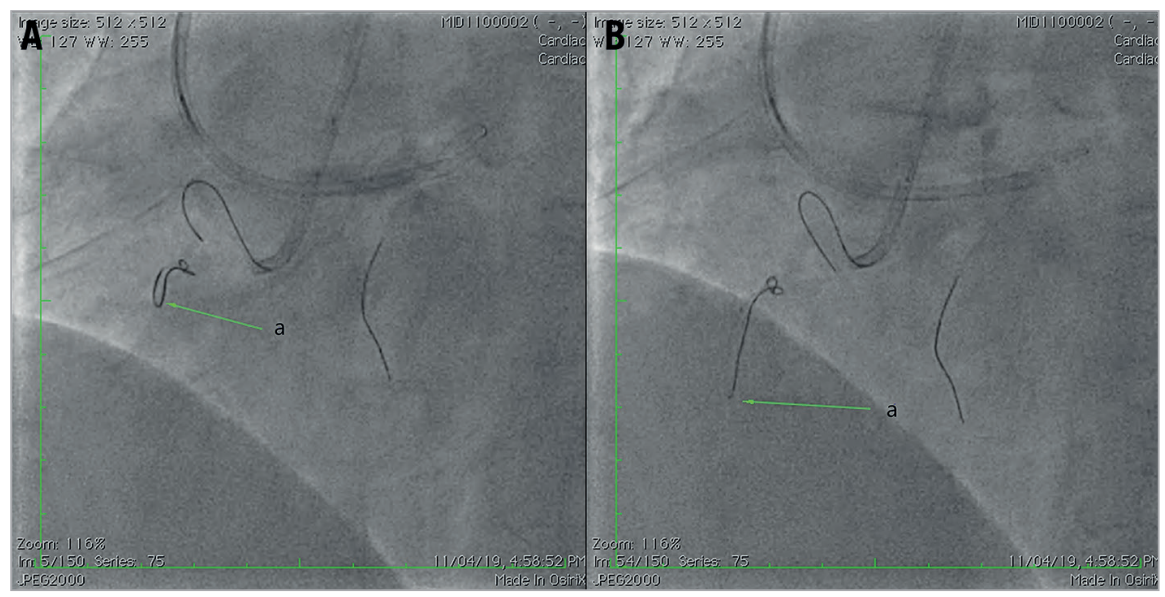

Figure 5. Knuckle technique. A) Knuckle in formation (arrow), B) Fully formed knuckle at the junction of a soft and stiff portion of the wire (arrow). 
the cap by other means ${ }^{11,12}$. Further, this allows entry of the microcatheter, through which a polymer jacketed wire is introduced, into the CTO body (Figure 6). Knuckling inside the lesion does not allow the subintimal space to get exposed to systemic pressure, as the entry is too narrow. Initially the proximal cap should be penetrated, if possible, using angiographic or intravascular ultrasound (IVUS) guidance and the knuckle technique is to be used if the antegrade wire escalation fails or when the antegrade wire gets trapped in plaque ${ }^{13}$. In the "long plus" type of CTO (i.e., longer CTO with added morphological complexities, such as calcification, tortuosity, and previous attempt with subintimal passage), if the antegrade technique is not progressing, the knuckle technique should be used with a clear-cut plan for re-entry. If the distal vessel is not accessible by retrograde channels, extreme care should be taken to perform reentry soon after exiting the CTO length.

\section{KNUCKLE PROXIMAL TO A CTO}

Knuckling proximal to a CTO is not ideal (Figure 7A, Figure 7B) due to direct exposure of the subintimal space to the systolic pressure of the aorta, leading to the expansion of the subintimal space, which may complicate further steps of re-entry (Figure 7C, Figure 7D). In such cases, measures to isolate the subintimal space from aortic pressure should be used, such as a TrapLiner ${ }^{\circledR}$ Catheter (Teleflex Medical, Wayne, PA, USA), proximal balloon occlusion, and/or deep seating of the guide.

In the presence of calcification and tortuosity, a large amount of force might be required, often utilising measures such as a large, deep-seated guide with microcatheter and anchor balloon (Figure 7). In cases of extreme resistance, a balloon (loaded on the knuckled wire) can be inflated in the vessel, just proximal to the occlusion, for maximal support ${ }^{12,14}$.

\section{Controlling knuckle size}

During knuckle wiring, it is preferable to keep the size of the knuckle as small as possible to minimise vessel trauma. Loops formed with a Fielder XT wire tend to be smaller than those formed with a Pilot 200 wire; as the stiffness of wire increases the diameter of the knuckle increases. The knuckle diameter can be controlled to some degree by adjusting the distance between the supporting microcatheter and the knuckle tip. Ultimately, however, the knuckle size is determined by the diameter of the vessel, and the proximity of the microcatheter to the knuckle tip determines the degree of force applied to create the dissection plane (Figure 8) ${ }^{12}$.

\section{Guide catheter support for the knuckle wire}

As the formation and advancement of the knuckle wire needs a forceful push to the guidewire, this requires sufficient guide catheter support ${ }^{15}$. In situations of inadequate guide support, the following techniques can be used to enhance guide support for knuckle wire advancement (Figure 9-Figure 11):

- anchor balloon

- balloon-assisted subintimal entry (BASE)/side-BASE with knuckle in impenetrable or ambiguous proximal cap. A monorail balloon with a size equivalent to the main vessel (BASE) or side branch (side-BASE) lumen diameter is inflated with a microcatheter on the side of the balloon; the knuckle wire is advanced through the microcatheter with the balloon inflated, which facilitates subintimal entry of the knuckle as well as giving good support for knuckle advancement.

- proximal over-the-wire balloon inflation with a knuckle-through. In cases where support enhancement is needed, and no option of a side branch anchor, an over-the-wire proximal balloon inflation with a knuckle-through can be used (Figure 11).

\section{When to use a knuckle wire KNUCKLE WIRE IN CTO PERCUTANEOUS CORONARY INTERVENTION (PCI)}

With the progress of wire technology, a knuckle wire should NOT be a first line wiring technique in most CTO lesions ${ }^{14,15}$.

1. A knuckle wire can be used when the vessel course is angiographically ambiguous, to reveal the vessel course (Figure 12).

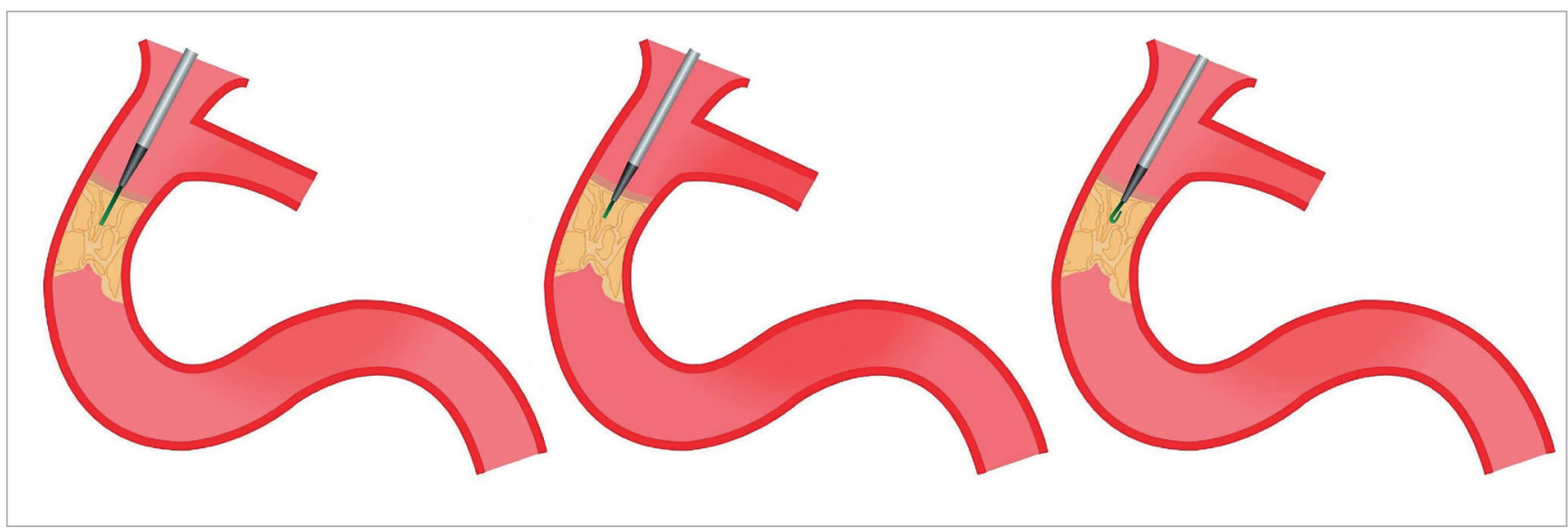

Figure 6. Penetration of proximal cap with a stiff wire to allow the introduction of microcatheter and soft wire into the CTO body. 

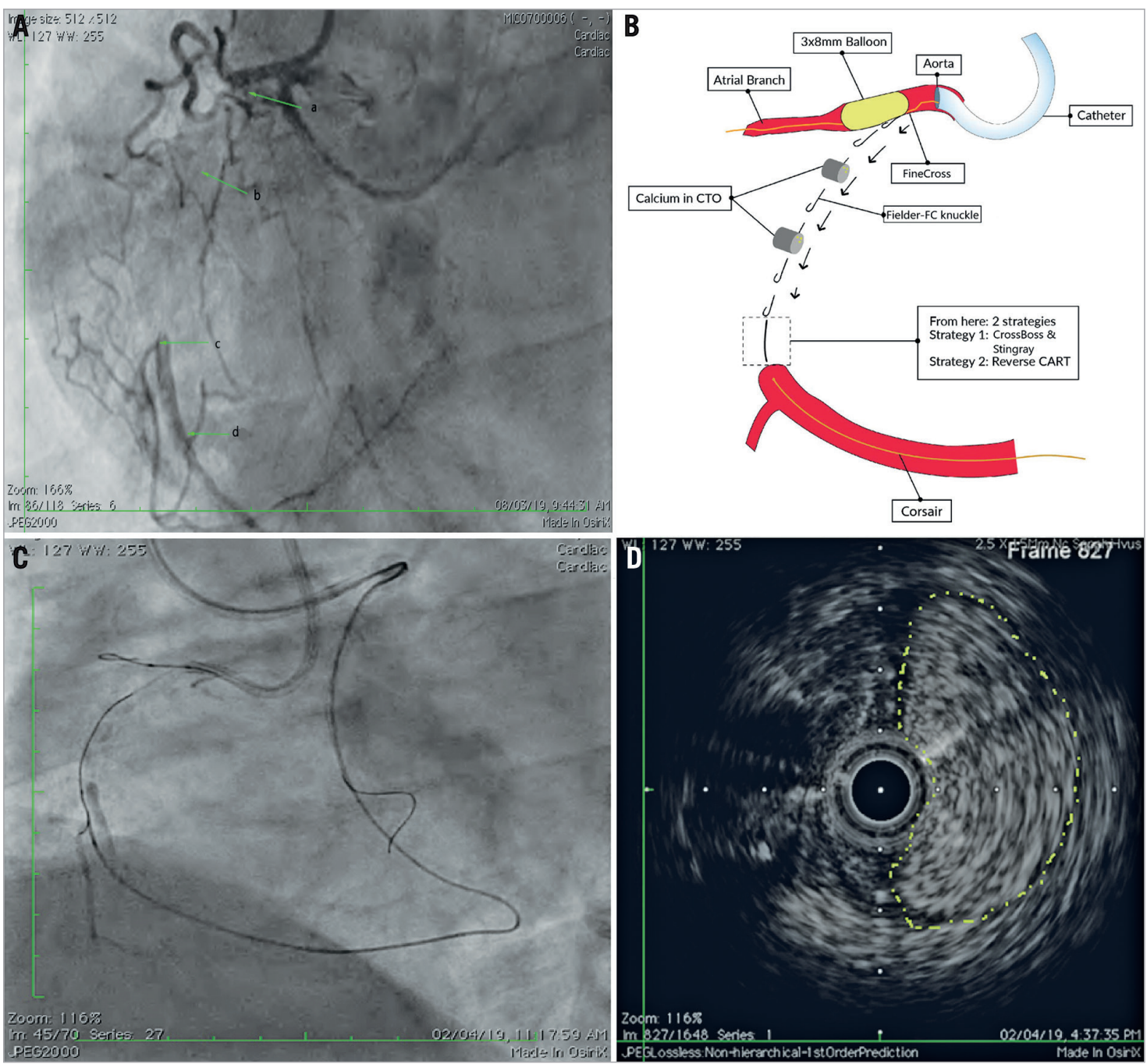

Figure 7. Knuckle proximal to CTO. A) Long RCA CTO with JCTO score 3 with impenetrable proximal cap. a: ambiguous proximal cap; b: calcium in the CTO; $c$ : bifurcation at CTO; d: good distal target with a lesion length of $>30 \mathrm{~mm}$. B) Knuckle wire made with side-BASE proximal to CTO. C) Subintimal haematoma created after knuckling, as subintimal space is directly exposed to aortic pressures. D) IVUS showing subintimal haematoma (yellow dotted outline). CTO: chronic total occlusion

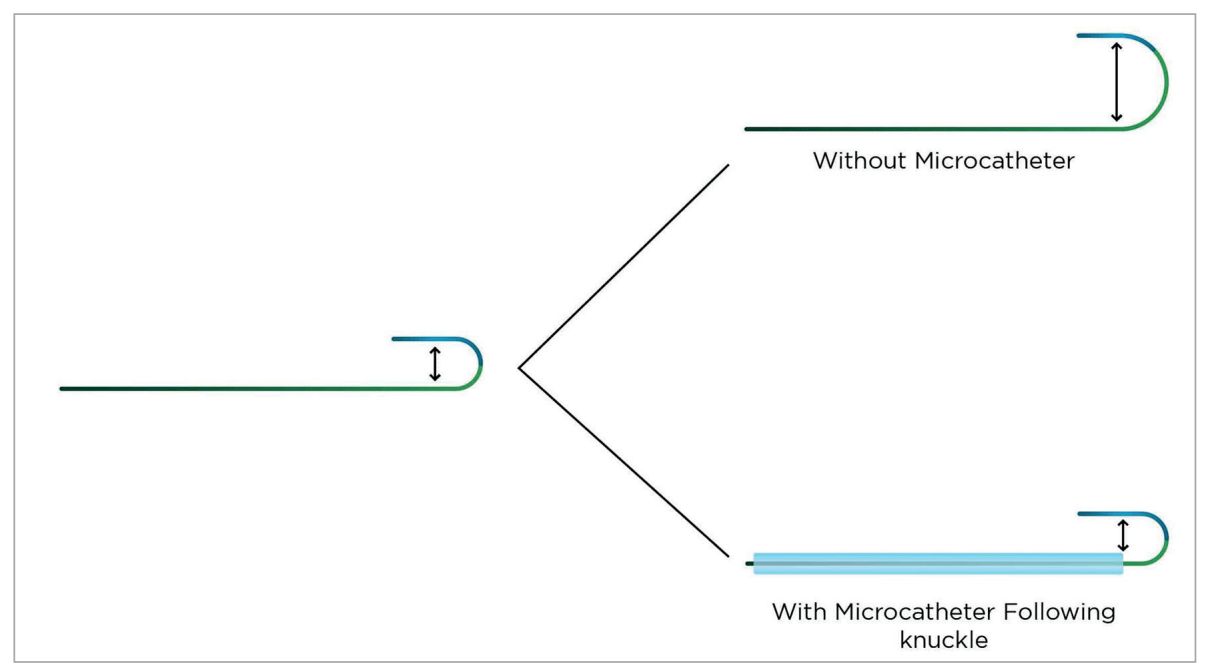

Figure 8. Controlling the knuckle. 


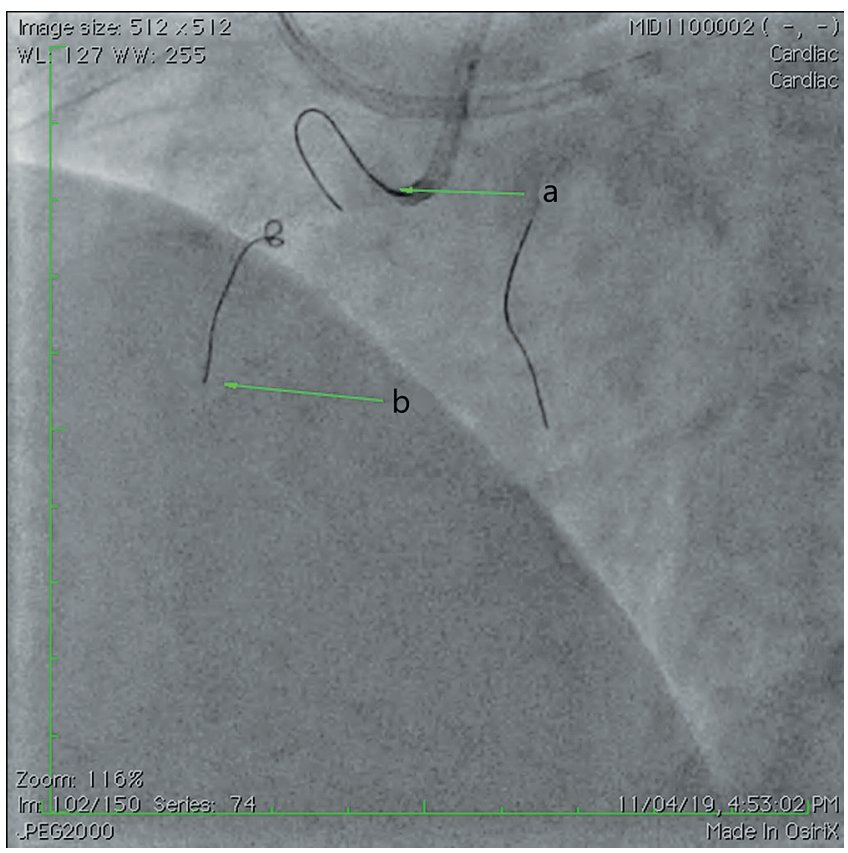

Figure 9. Proximal RCA CTO. Knuckle pushed with an anchor balloon in the atrial branch, a: anchor balloon $(1.5 \times 12 \mathrm{~mm})$; b: knuckle with a Fielder XT-R.

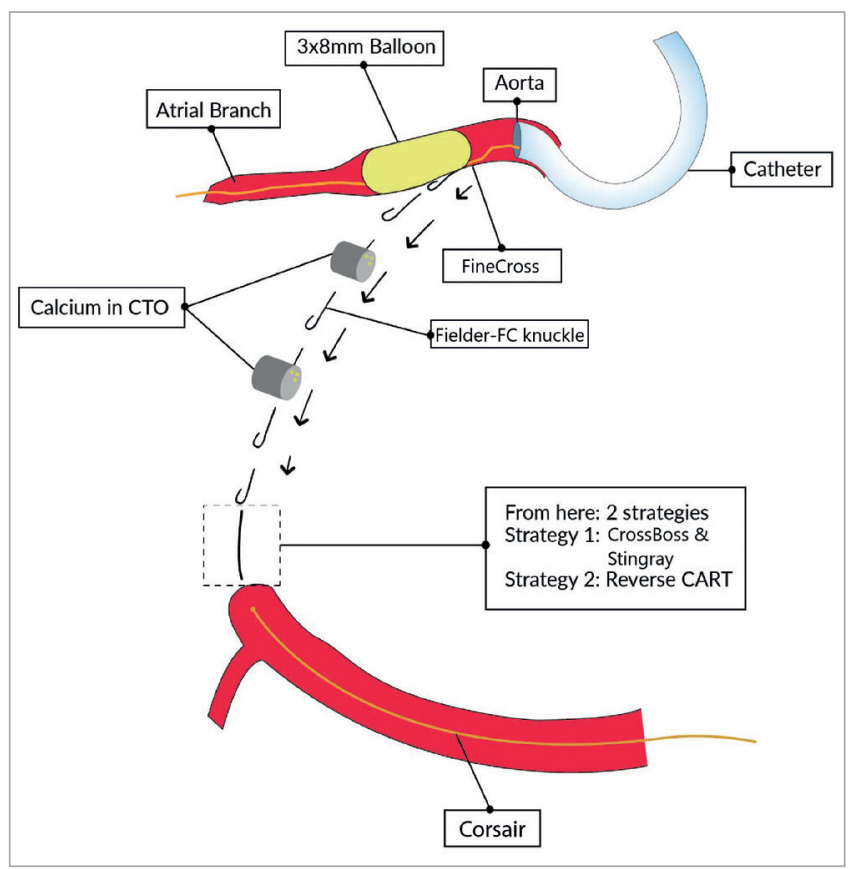

Figure 10. BASE/side-BASE with knuckle in impenetrable or ambiguous proximal cap

2. In highly calcified and tortuous long CTO, where high penetration wiring may perforate the vessel, a knuckle wire can be considered (Figure 13). The angiographic representation of knuckle wire progression is as shown in Figure 14.

3. A knuckle wire can be used after difficult and long retrograde channel crossing of a long CTO, where the procedure time is

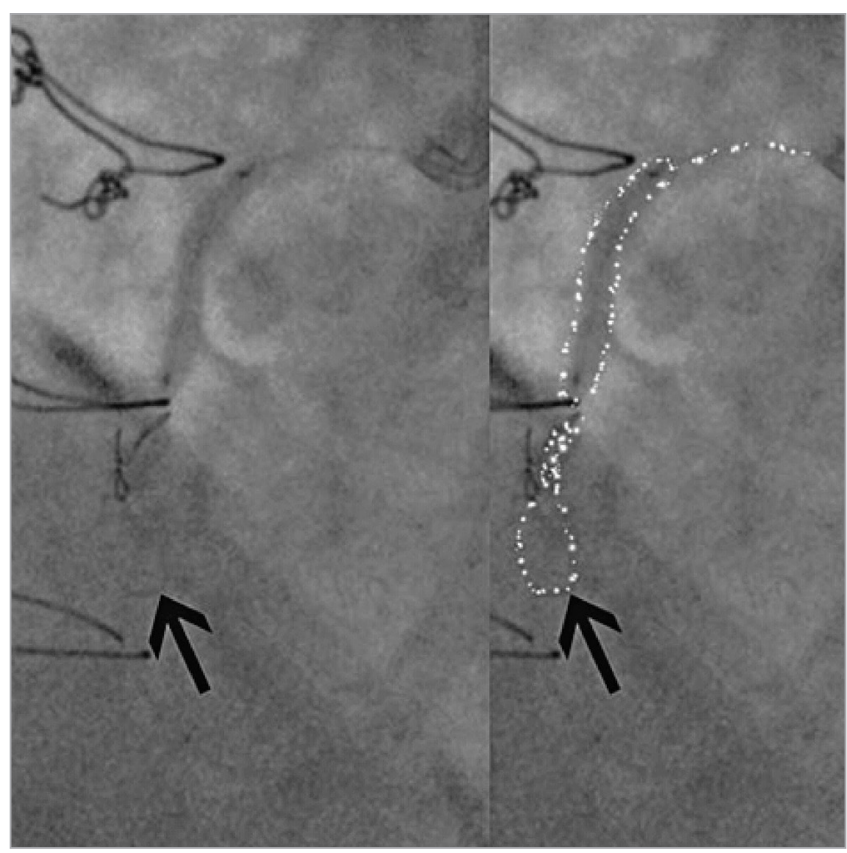

Figure 11. Knuckle formation with support of a proximal OTW inflated balloon.

long and the radiation limit has been nearly reached. In this situation, a knuckle wire can rapidly cross the CTO body allowing completion of the case before the radiation limit is reached.

4. After the failure of antegrade/retrograde wire escalation, or failure of the retrograde approach.

5. A knuckle wire can be used in conjunction with a CrossBoss catheter to facilitate the crossing of tortuous segments (Figure 14), avoid side branch entry (Figure 15), and circumvent calcium spots stalling the CrossBoss catheter (Figure 16).

6. Rarely, knuckling can be used as an initial crossing strategy (primary dissection/re-entry), providing that the lesion fulfils the following characteristics (Figure 17) ${ }^{16}$ :

- well-defined proximal cap

$-\geq 20 \mathrm{~mm}$ length

- large calibre distal vessel

- no large branches within the CTO or, more importantly, at the distal cap.

7. Knuckling in retrograde $\mathrm{PCI}^{17}$ can be used in the following circumstances:

- ambiguous course in CTO

- tortuous CTO segment

- heavy calcification.

\section{Knuckle wire vs CrossBoss for subintimal tracking}

Although, the knuckle technique is a faster and less expensive technique of subintimal tracking, it is not totally controllable and predictable. Re-entry can get complicated due to wider dissection (Figure 18A) or an intramural haematoma (Figure 18B, Figure 18C) compressing the true lumen. In contrast, the CrossBoss catheter 

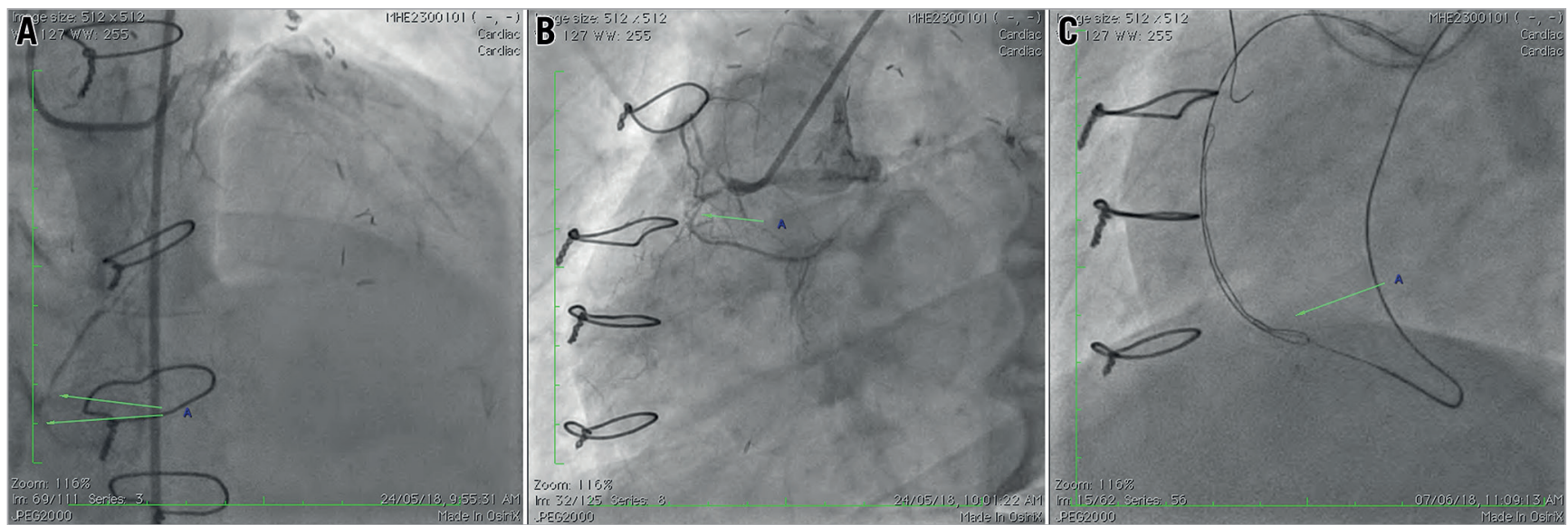

Figure 12. Knuckle in CTO PCI. A) Proximal RCA CTO with calcium in a post CABG patient with JCTO score of 3, with PLVB filling from left system (arrows). B) Distal RCA filling up to crux in retrograde injection; course of RCA is invisible with an angle $>40$, arrow indicates proximal RCA CTO. C) Antegrade knuckle to guide and navigate retrograde wire, with antegrade knuckle guiding retrograde wire (arrow).

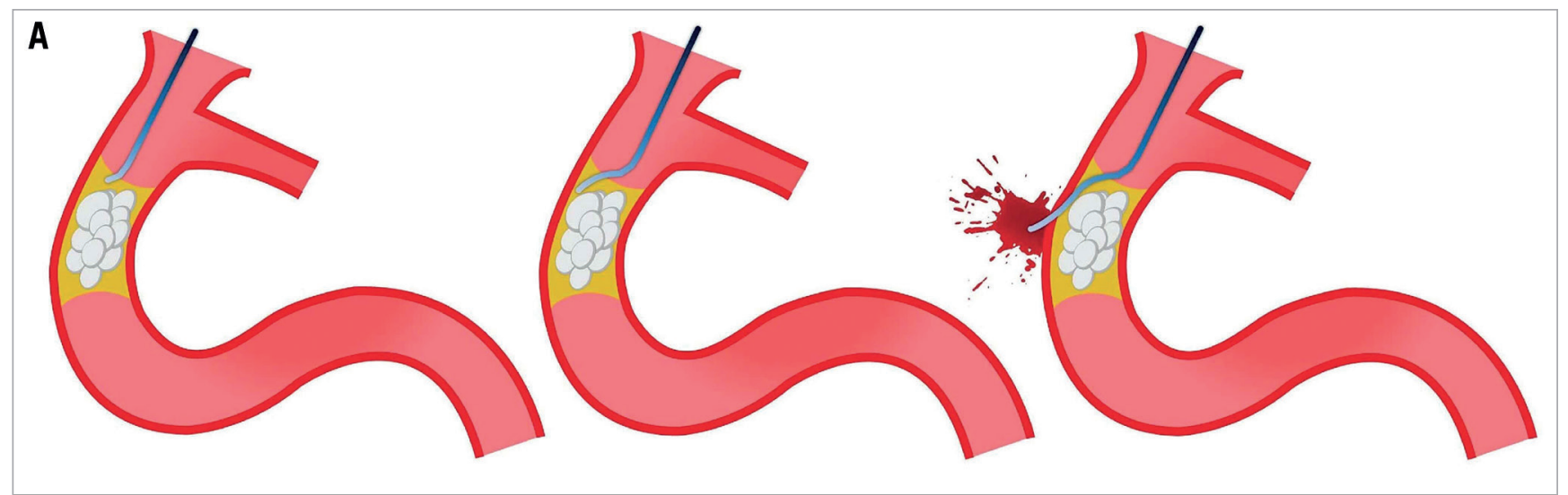

B
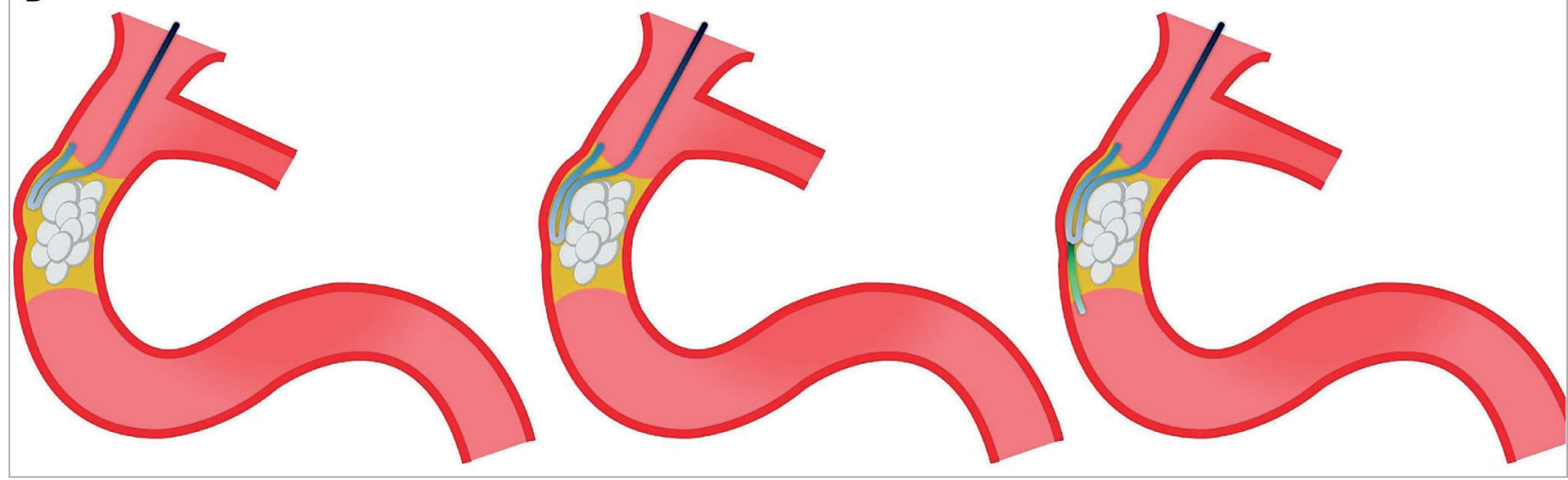

Figure 13. Progression of knuckle wire though highly calcified and long tortuous CTO. A) Perforation of the vessel with the use of a stiff wire. B) Use of a soft wire will prevent perforation.

acts like a microknuckle with its $1 \mathrm{~mm}$ blunt, stainless steel tip causing highly controllable microdissection and rarely complicating re-entry into the true lumen (Figure 18D, Figure 18E). Stent malapposition is frequently observed in knuckled areas due to deeper shelving of the subintimal space (Figure 19A) with knuckle; however, it is rarely seen with the CrossBoss catheter subintimal dissection. Therefore, a CrossBoss catheter with Stingray LP system provides a more controllable, predictable and small subintimal dissection with a high probability of successful re-entry and low possibility of a malapposed stent than 


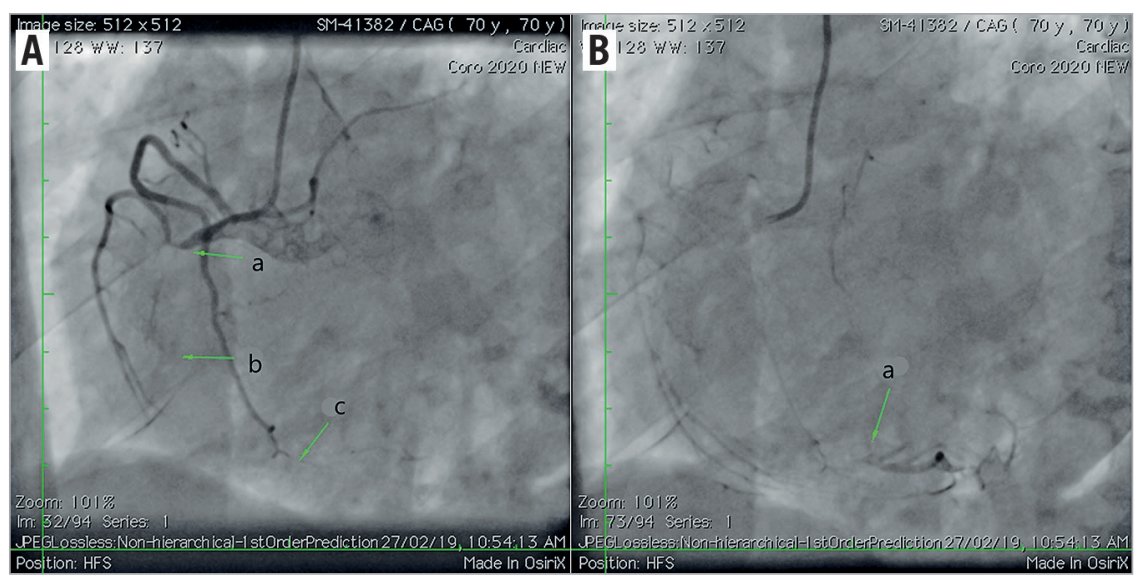

Figure 14. Highly calcified and long tortuous CTO. A) Clearly visible proximal cap (a), lesion length $>50 \mathrm{~mm}$ (b), and angulation $>40^{\circ}$ (c). (B) Good distal target with a bifurcation at the distal cap (a).
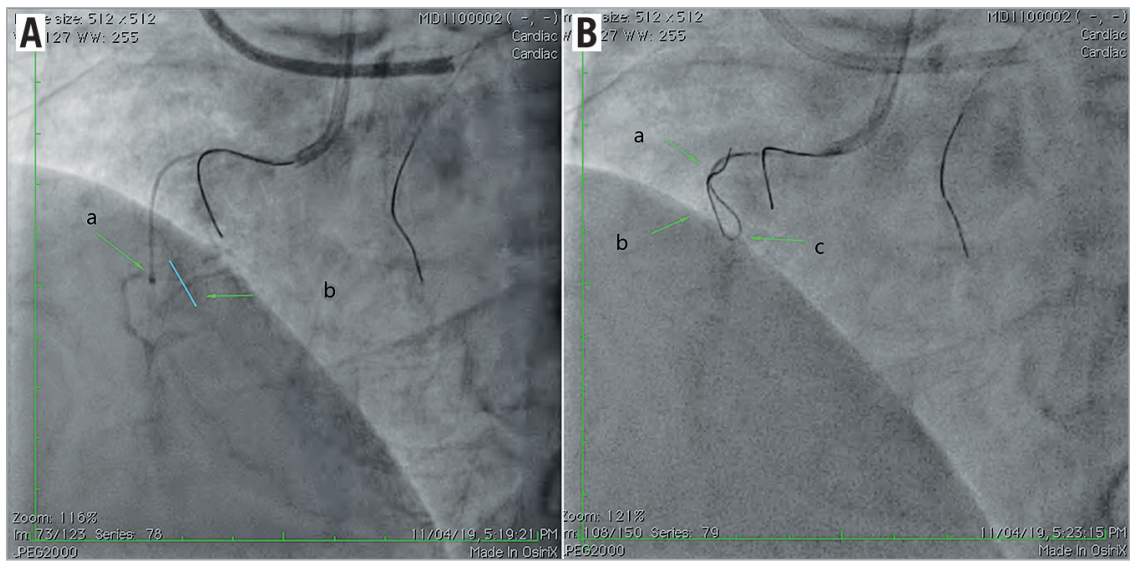

Figure 15. Knuckle used in conjunction with a CrossBoss catheter to avoid side branch entry. A) CrossBoss in side-branch (a) and axis of the main vessel RCA (b). B) Knuckle to cross side-branch for CrossBoss advancement beyond branch point; a) indicates CrossBoss, b) indicates location of side-branch, and c) indicates knuckle with Pilot 150.
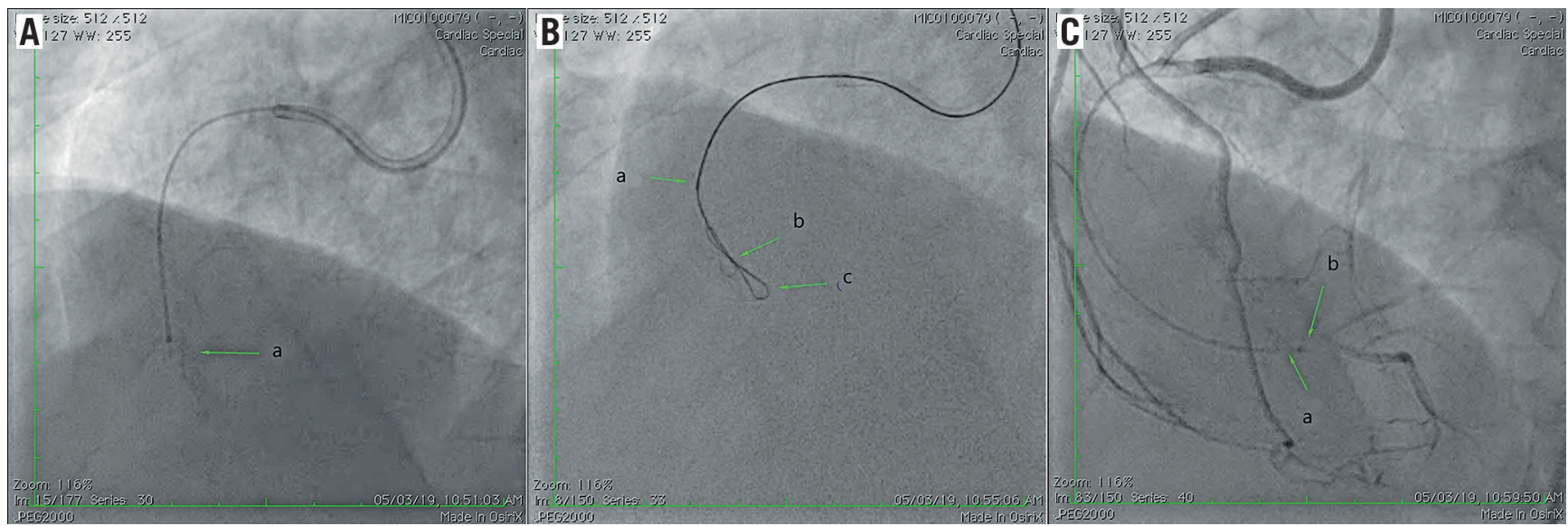

Figure 16. Knuckle used in conjunction with CrossBoss to cross the circumvent calcium spots. A) CrossBoss stuck in lesion, where a) indicates the CrossBoss stalled by calcium in the mid-CTO. B) Knuckle wire to cross the calcium and CrossBoss advanced over knuckle wire, a: retracted CrossBoss; b: point where CrossBoss is stalled; : Fielder-FC knuckle crossed the point at which CrossBoss stalled. C) CrossBoss advancement to re-entry zone. a: CrossBoss; $b$ : distal RCA lumen. 


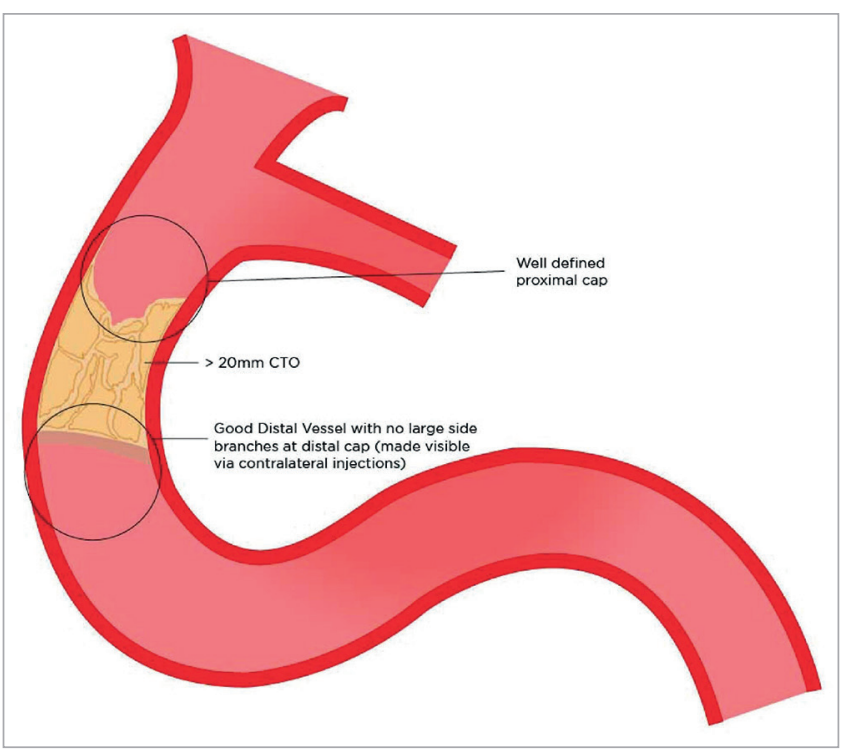

Figure 17. Criteria for using knuckle as an initial crossing strategy. knuckling (Figure 19B). The principle of minimum vessel distortion should be applied and it is preferable that the majority of subintimal tracts be created by a CrossBoss catheter rather than a knuckle wire, where possible $e^{5-7}$.

\section{Re-entry after knuckle technique}

The initial description of knuckle wire use in coronary CTO PCI was the STAR technique, in which the knuckle wire was used for subintimal tracking as well as re-entry into the true lumen, which is unpredictable, and unsuccessful in a significant number of cases. In present day CTO practice, the knuckle wire is used in conjunction with the CrossBoss-Stingray catheter for more predictable re-entry ${ }^{2,15,18,19}$. Stingray is a modified hydrophiliccoated balloon with a flat profile. It has dual lumen with $180^{\circ}$ opposed and offsetting exit ports enabling selective guidewire re-entry.

Re-entry can also be performed with mini-STAR or limited antegrade subintimal tracking (Figure 20) ${ }^{11,20-22}$.
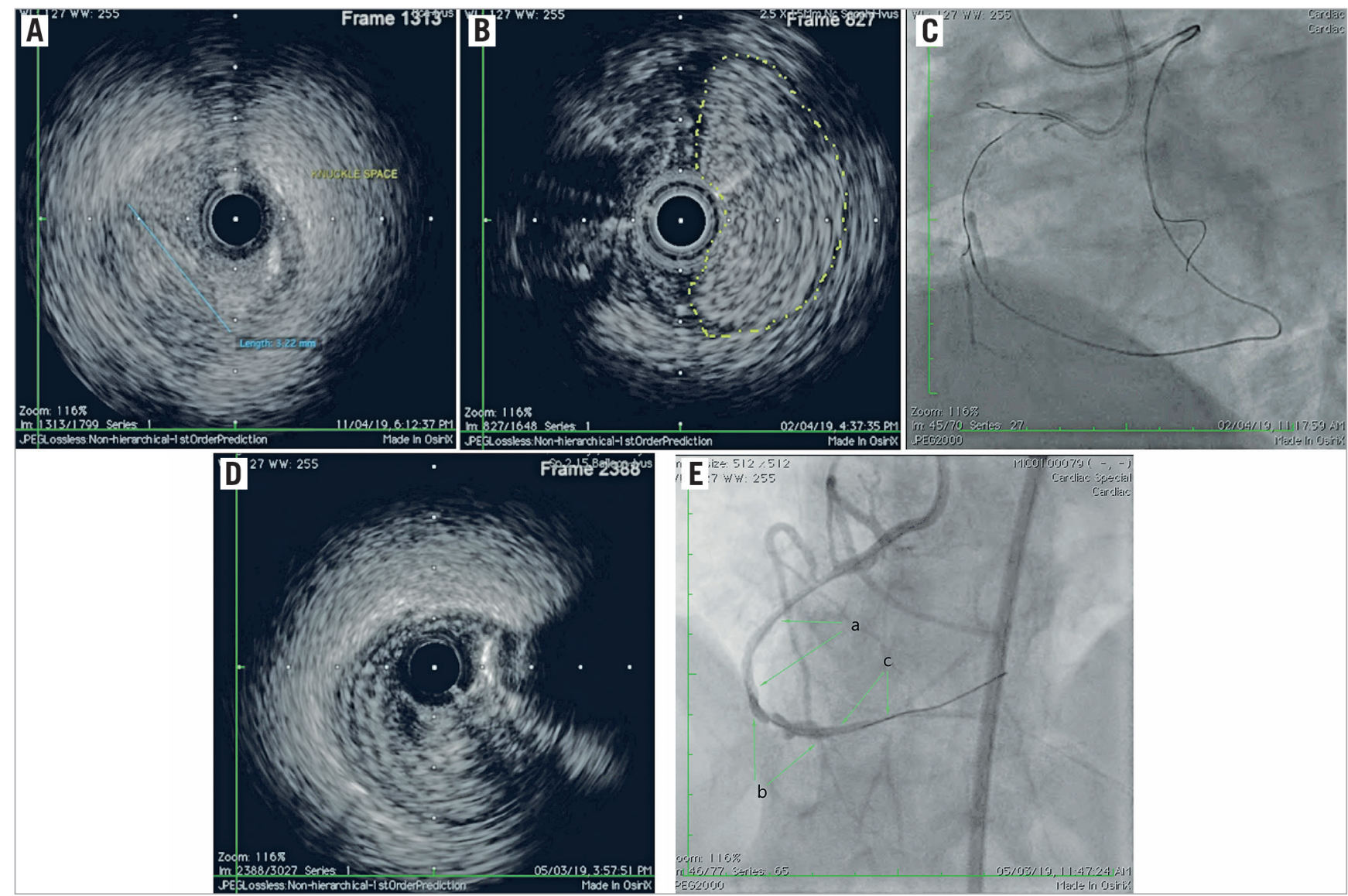

Figure 18. Knuckle wire versus CrossBoss for subintimal tracking. A) IVUS showing wide crescent-shaped subintimal space created by knuckle. B) Intramural haematoma (yellow dotted outline) created by knuckle in distal RCA. C) Intramural haematoma in distal RCA, post knuckling. D) IVUS showing small, circular subintimal space created by CrossBoss. E) Fluoroscopy showing wide irregular lumen in knuckled area and regular, uniform lumen in CrossBoss area. a: area of CrossBoss advancement; $b$ : knuckled area of the vessel; $c$ : area of CrossBoss advancement. 


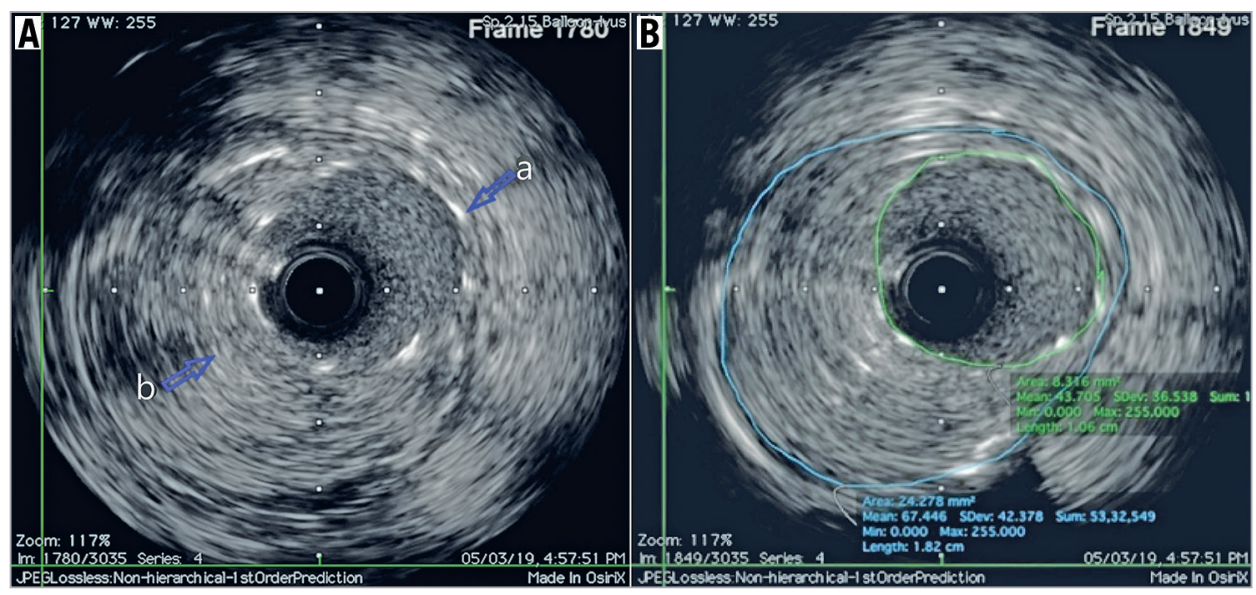

Figure 19: CrossBoss with Stingray to overcome stent malapposition. A) Stent malapposition between 3 and 6 o'clock in the knuckled arterial segment. a: stent strut; $b$ : vessel wall. B) Proper stent apposition in CrossBoss arterial segment.

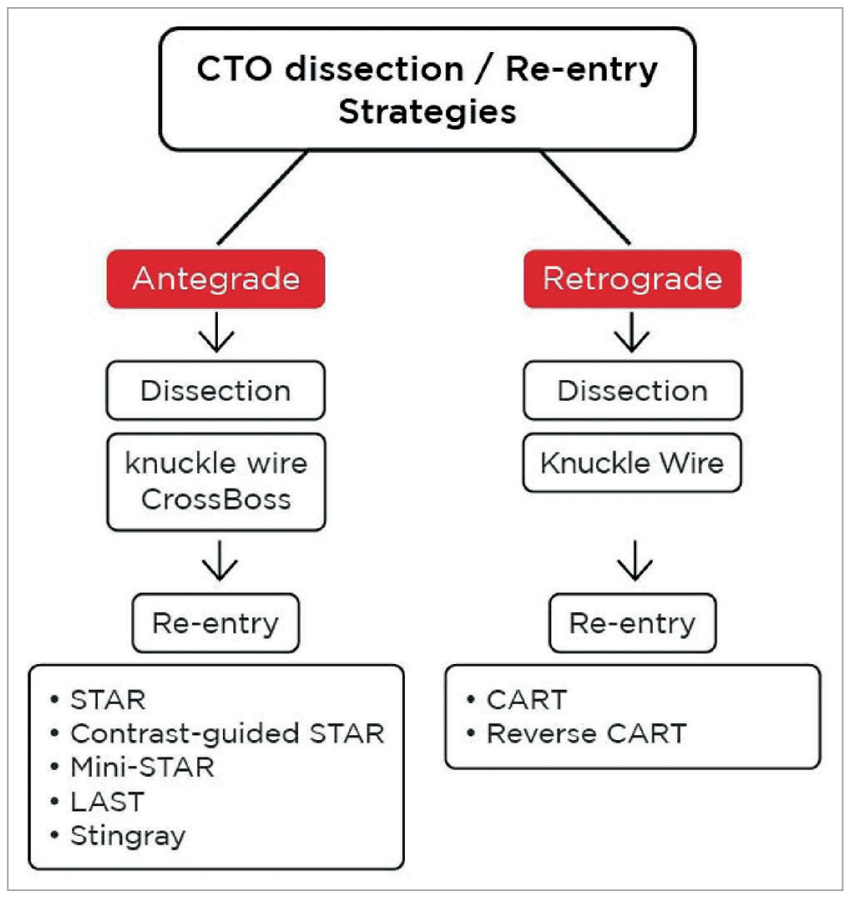

Figure 20. CTO dissection/re-entry strategies after knuckle wiring.

\section{When not to perform a stand-alone knuckle wire technique}

The biggest challenge for the knuckle procedure is the unpredictable re-entry, which can be avoided by careful case selection. Presence of good interventional collaterals and access to retrograde gear is always a big advantage when performing the knuckle technique as this ensures the safety of the distal vessel if the knuckle does not create the desired result:

- when the proximal/distal cap is over a major bifurcation point

- ambiguous CTO entry cap, when cap penetration is not possible with coronary angiography/IVUS guidance
- when the CTO body involves a significant side branch

- when there is a diffuse distal disease, which may preclude a predictable re-entry.

\section{Conclusion}

The knuckle wire technique in its conventional form, or in the various fine-tuned modifications that provide "controlled knuckle", add to the success of CTO intervention with ambiguous vessel course and long length occlusions. Often it is attempted as a last resort when intervention is neither progressing antegradely or retrogradely due to impenetrable plaque or ambiguous course. In the contemporary CTO PCI scenario, antegrade knuckling is predominantly used to facilitate CrossBoss catheter advancement and further re-entry with the Stingray catheter. However, the technique requires a thorough understanding of the CTO anatomy and orientation of the subintimal space created. A clear-cut strategy to protect the distal territory from subintimal haematoma is of paramount importance while performing this procedure.

\section{Conflict of interest statement}

The authors have no conflicts of interest to declare.

\section{References}

1. Colombo A, Mikhail GW, Michev I, Iakovou I, Airoldi F, Chieffo A, Rogacka R, Carlino M, Montorfano M, Sangiorgi GM, Corvaja N, Stankovic G. Treating chronic total occlusions using subintimal tracking and re-entry: the STAR technique. Catheter Cardiovasc Interv. 2005;64:407-11.

2. Martinez-Rumayor AA, Banerjee S, Brilakis ES. Knuckle wire and stingray balloon for recrossing a coronary dissection after loss of guidewire position. JACC Cardiovasc Interv. 2012;5:e31-2.

3. Tasic M, Sreckovic MJ, Jagic N, Miloradovic V, Nikolic D. Knuckle technique guided by intravascular ultrasound for in-stent restenosis occlusion treatment. Postepy Kardiol Interwencyjnej. 2015;11:58-61. 
4. Mishra S. Unraveling the mystique of CTO Interventions: tips and techniques of using hardware to achieve success. Indian Heart J. 2017;69:266-76.

5. Brilakis ES, Karmpaliotis D, Vo MN, Garcia S, Michalis L, Alaswad K, Doshi P, Lombardi WL, Banerjee S. Advances in the management of coronary chronic total occlusions. $J$ Cardiovasc Transl Res. 2014;7:426-36.

6. Harding SA, Wu EB, Lo S, Lim ST, Ge L, Chen JY, Quan J, Lee SW, Kao HL, Tsuchikane E. A new algorithm for crossing chronic total occlusions from the Asia Pacific Chronic Total Occlusion Club. JACC Cardiovasc Interv. 2017;10:2135-43.

7. Rinfret S, Ybarra LF. Antegrade Chronic Total Occlusion Crossing: CrossBoss First or Last? JACC Cardiovasc Interv. 2018; 11:234-6.

8. Isawa T, Ochiai M, Munehisa M, Ootomo T. A Novel, Modified Reverse Controlled Antegrade and Retrograde Subintimal Tracking Technique for Bypassing the Calcified Proximal Cap of Coronary Total Occlusions. Case Rep Cardiol. 2017;2017:3850646.

9. Lim MC. Antegrade techniques for chronic total occlusions. Curr Cardiol Rev. 2015;11:285-90.

10. Carlino M, Demir OM, Colombo A, Azzalini L. Microcatheter knuckle technique: a novel technique for negotiating the subintimal space during chronic total occlusion recanalization. Catheter Cardiovasc Interv. 2018;92:1256-60.

11. Dash D. Guidewire crossing techniques in coronary chronic total occlusion intervention: A to Z. Indian Heart J. 2016;68: 410-20.

12. Wilson W, Spratt JC. Advances in procedural techniques-antegrade. Curr Cardiol Rev. 2014;10:127-44.

13. Shaukat A, Mooney M, Burke MN, Brilakis ES. Use of chronic total occlusion percutaneous coronary intervention techniques for treating acute vessel closure. Catheter Cardiovasc Interv. 2018; 92:1297-300.

14. Danek BA, Karatasakis A, Karmpaliotis D, Alaswad K, Yeh RW, Jaffer FA, Patel M, Bahadorani J, Lombardi WL, Wyman MR, Grantham JA, Doing A, Moses JW, Kirtane A, Parikh M, Ali ZA, Kalra S, Kandzari DE, Lembo N, Garcia S, Rangan BV, Thompson CA, Banerjee S, Brilakis ES. Use of antegrade dissection re-entry in coronary chronic total occlusion percutaneous coronary intervention in a contemporary multicenter registry. Int J Cardiol. 2016;214:428-37.

15. Wu EB, Tsuchikane E, Lo S, Lim ST, Ge L, Chen JY, Qian J, Lee SW, Harding S, Kao HL. Retrograde algorithm for chronic total occlusion from the Asia Pacific Chronic Total Occlusion club. AsiaIntervention. 2018;4:98-107.

16. Joyal D, Thompson CA, Grantham JA, Buller CE, Rinfret S. The retrograde technique for recanalization of chronic total occlusions: a step-by-step approach. JACC Cardiovasc Interv. 2012;5: $1-11$.

17. Brilakis ES, Badhey N, Banerjee S. "Bilateral knuckle" technique and Stingray re-entry system for retrograde chronic total occlusion intervention. J Invasive Cardiol. 2011;23:E37-9.

18. Schumacher SP, Stuijfzand WJ, Opolski MP, van Rossum AC, Nap A, Knaapen P. Percutaneous coronary intervention of chronic total occlusions: when and how to treat. Cardiovasc Revasc Med. 2019;20:513-22.

19. Tarar MN, Christakopoulos GE, Brilakis ES. Successful management of a distal vessel perforation through a single 8 French guide catheter: Combining balloon inflation for bleeding control with coil embolization. Catheter Cardiovasc Interv. 2015;86: 412-6.

20. Michael TT, Papayannis AC, Banerjee S, Brilakis ES. Subintimal dissection/re-entry strategies in coronary chronic total occlusion interventions. Circ Cardiovasc Interv. 2012;5:729-38.

21. Maeremans J, Dens J, Spratt JC, Bagnall AJ, Stuijfzand W, Nap A, Agostoni P, Wilson W, Hanratty CG, Wilson S, Faurie B, Avran A, Bressollette E, Egred M, Knaapen P, Walsh S; RECHARGE Investigators. Antegrade dissection and re-entry as part of the hybrid chronic total occlusion revascularization strategy: asubanalysis of the RECHARGE Registry (Registry of CrossBoss and Hybrid Procedures in France, the Netherlands, Belgium and United Kingdom). Circ Cardiovasc Interv. 2017;10:e04791.

22. Godino C, Sharp AS, Carlino M, Colombo A. Crossing CTOsThe tips, tricks, and specialist Kit that can mean the difference between success and failure. Catheter Cardiovasc Interv. 2009; 74:1019-46. 